\title{
ON STRONG SOLUTIONS OF THE DIFFERENTIAL EQUATIONS MODELING THE STEADY FLOW OF CERTAIN INCOMPRESSIBLE GENERALIZED NEWTONIAN FLUIDS
}

\author{
M. BILDHAUER, M. FUCHS, AND X. ZHONG
}

\begin{abstract}
A system of nonautonomous partial differential equations describing the steady flow of an incompressible fluid is considered. The existence of a strong solution of that system is proved under suitable assumptions on the data. In the 2D-case this solution turns out to be of class $C^{1, \alpha}$.
\end{abstract}

\section{$\S 1$. INTRODUCTION}

Our purpose in this paper is to establish the existence of a so-called strong solution for a system of equations that can be viewed as a modification and an extension of the classical stationary Navier-Stokes system to the case of certain incompressible generalized Newtonian fluids of anisotropic power-law type. The main new feature is that we allow the viscous part of the Cauchy stress tensor $T$ to depend also on the spatial variable $x$, which is not a trivial extension of the results obtained in $\mathrm{ABF}$ for the autonomous anisotropic case. Even if the dependence on $x$ is smooth, we are confronted with more serious restrictions on the range of anisotropy than in $\mathrm{ABF}$. This is not a consequence of technical inability - the reason is some kind of Lavrentiev phenomenon explained in the setting of variational problems in the paper [ELM] and also discussed in BF4. We want to give a more detailed comment: if we consider a variational problem of the form

$$
J[u]=\int_{\Omega} f(x, \nabla u) \mathrm{d} x \rightarrow \min
$$

with integrand $f$ of anisotropic $(p, \bar{q})$-growth (see (1.2) and (1.3)), then the existence of a minimizer $u$ for suitable boundary data can easily be established in the energy class $W_{p}^{1} \cap[J<\infty]$ with the help of the direct method. But as outlined in [ELM], this unique solution may fail to have any higher integrability properties if the exponents $p$ and $\bar{q}$ are not related through condition (1.5) from below; i.e., in the example of [ELM] (which was inspired by [Z] ) a gap phenomenon occurs, and up to now it is unclear if (1.5) is a sufficient condition for excluding the gap phenomenon. In contrast to this situation, Lavrentiev's phenomenon for the autonomous anisotropic case does not occur merely under the weaker condition $\bar{q}<p(1+2 / n)$ (see the comments in [ELM] and [BF4]).

Now, in the setting of fluids, we are not going to minimize a variational integralinstead of this we want to show that the underlying system of nonlinear partial differential equations modelling our stationary flow has a solution with some degree of smoothness; for example, the velocity field should solve the problem almost everywhere, and in the $2 \mathrm{D}$-case the solution we like to construct should be a (more or less) classical one. In the papers $\mathrm{ABF}, \mathrm{BF} 1, \mathrm{BF}$ ], and $\mathrm{BFZ}$ we studied this question for the autonomous

2000 Mathematics Subject Classification. Primary 76M30, 76B03, 35Q35.

Key words and phrases. Generalized Newtonian fluids, anisotropic dissipative potentials, existence and regularity of solutions. 
case and showed the existence of regular solutions under the same condition for the range of anisotropy as in the variational setting. But as outlined above, the stronger condition (1.5) seems to be necessary to provide some hope to reach our goal in the nonautonomous case; otherwise there might only exist weak solutions with no additional smoothness properties.

In contrast to the variational setting, it turns out that, actually, (1.5) is sufficient for proving the existence of a good solution of the problem under consideration. In fact, Esposito, Leonetti, and Mingione showed in Section 5 of [ELM that (1.5) implies at least the regularity of the weak solution of a certain relaxed minimization problem, and we proved in BF4 that this minimizer is also a solution of the Euler-Lagrange equation of the starting minimization problem (but not necessarily a minimizer). In the present paper we adopt this technique and establish the existence of rather smooth solutions for nonautonomous anisotropic flows.

Let us now fix our assumptions. Suppose that we are given a bounded Lipschitz domain $\Omega \subset \mathbb{R}^{n}, n=2,3$, together with a system $g: \Omega \rightarrow \mathbb{R}^{n}$ of volume forces whichfor technical simplicity - is assumed to be of class $L^{\infty}\left(\Omega ; \mathbb{R}^{n}\right)$. Then we are looking for a velocity field $u$ : $\Omega \rightarrow \mathbb{R}^{n}$ together with a pressure function $\pi: \Omega \rightarrow \mathbb{R}$ such that the following nonautonomous system of partial differential equations is satisfied:

$$
\left\{\begin{array}{r}
-\operatorname{div}\{T(\cdot, \varepsilon(u))\}+\nabla \pi+[\nabla u] u=g \quad \text { in } \Omega, \\
\operatorname{div} u=0 \quad \text { in } \Omega, \quad u=0 \quad \text { on } \partial \Omega .
\end{array}\right.
$$

Here $\varepsilon(u)$ denotes the symmetric gradient of $u$, i.e., $\varepsilon(u)=\frac{1}{2}\left(\nabla u+\nabla u^{T}\right)$, and $[\nabla u] u$ is the convective term $u^{k} \frac{\partial u}{\partial x_{k}}$ (summation with respect to $k$ ). The tensor-valued function $T=T(x, \varepsilon)$ is defined for all $x \in \bar{\Omega}$ and all matrices $\varepsilon \in \mathbb{S}^{n}(:=$ space of symmetric $(n \times n)$-matrices) and arises as the gradient with respect to the second argument of a smooth convex potential $f=f(x, \varepsilon)$. More precisely, we impose the following conditions on the potential $f$. With exponents $1<p \leq \bar{q}<\infty$ and constants $\lambda, \Lambda, c_{1}>0$, the energy density $f: \bar{\Omega} \times \mathbb{S}^{n} \rightarrow[0, \infty)$ satisfies the inequalities

$$
\begin{aligned}
\lambda\left(1+|\varepsilon|^{2}\right)^{\frac{p-2}{2}}|\sigma|^{2} \leq D_{\varepsilon}^{2} f(x, \varepsilon)(\sigma, \sigma) & \leq \Lambda\left(1+|\varepsilon|^{2}\right)^{\frac{\bar{q}-2}{2}}|\sigma|^{2}, \\
\left|D_{x} D_{\varepsilon} f(x, \varepsilon)\right| & \leq c_{1}\left(1+|\varepsilon|^{2}\right)^{\frac{\bar{q}-1}{2}}
\end{aligned}
$$

for all $x \in \bar{\Omega}$ and all $\varepsilon, \sigma \in \mathbb{S}^{n}$. Here we assume that all the partial derivatives occurring in (1.2) and (1.3) are at least continuous functions. The reader should note that (1.2) implies the anisotropic growth condition

$$
a|\varepsilon|^{p}-b \leq f(x, \varepsilon) \leq A|\varepsilon|^{\bar{q}}+B
$$

with suitable positive constants $a, A, b$, and $B$. Now we can state our existence and regularity result concerning system (1.1).

Theorem 1.1. Suppose that (1.2) and (1.3) are satisfied together with

$$
p> \begin{cases}6 / 5 & \text { if } n=2, \\ 9 / 5 & \text { if } n=3 .\end{cases}
$$

Suppose further that

$$
\bar{q}<p\left(1+\frac{1}{n}\right)
$$

i) Then there exists a velocity field $u$ of class $\stackrel{\circ}{W}{ }_{p}^{1} \cap W_{t, \mathrm{loc}}^{2}\left(\Omega ; \mathbb{R}^{n}\right)$ for some $t>1$ and a pressure function $\pi$ of class $W_{s, \mathrm{loc}}^{1}(\Omega)$ for some $s>1$ such that (1.1) is satisfied almost everywhere. 
ii) If $n=2$ and if in addition $\bar{q}<p+2$, then the first derivatives of $u$ are Hölder continuous functions in $\Omega$.

Remark 1.1. At the end of $\$ 2$ we shall show that we can choose $t=2$ provided that $p \geq 2$. If $p<2$, then any number

$$
t< \begin{cases}2 & \text { if } n=2 \\ \frac{3 p}{p+1} & \text { if } n=3\end{cases}
$$

is admissible. Moreover, we shall establish that $D_{\varepsilon} f(\cdot, \varepsilon(u))$ belongs to the class

$W_{\frac{q}{(q-1)}, \text { loc }}^{1}\left(\Omega ; \mathbb{S}^{n}\right)$. Therefore, some bound for the exponent $s$ can be calculated with the help of equation (1.1).

Let us compare Theorem 1.1 to the known results.

i) A very general existence result for the nonautonomous isotropic case, i.e., the case where $p=\bar{q}$, was obtained in [FMS] by Frehse, Málek, and Steinhauer, even without the assumption that the tensor $T(x, \varepsilon)$ is generated by a potential $f$. Moreover, they replaced (1.4) by the requirement that $p>2 n /(n+2)$. But the solution they obtained is merely a weak solution, i.e., it belongs to the space $\stackrel{\circ}{W}_{p}^{1}\left(\Omega ; \mathbb{R}^{n}\right)$ and satisfies (1.1) in the distributional sense.

ii) The isotropic autonomous case, where $T=T(\varepsilon)$ and $T=D f$ with potential $f$ of $p$-growth, was discussed in the paper [KMS] by Kaplický, Málek, and Stará. They considered planar flows and proved the existence of a smooth velocity field $u$ under condition (1.4).

iii) In the papers $[\mathrm{BF} 1, \mathrm{ABF}, \mathrm{BF}$ ], and $[\mathrm{BFZ}$ the autonomous anisotropic case was investigated with the result that Theorem 1.1 is true under the weaker condition $\bar{q}<p(1+2 / n)$. At this stage we would like to emphasize again that the occurrence of the variable $x$ in the potential $f$ is not only a technicality. As was shown by Esposito, Leonetti, and Mingione [ELM], in the setting of variational problems a Lavrentiev phenomenon must be expected, which means that even in dimension 2 , singular solutions may occur if the condition $\bar{q}<3 p / 2$ is violated, whereas for the autonomous $2 \mathrm{~d}$-case the condition $\bar{q}<2 p$ is sufficient for regularity. For a further discussion, we refer to the paper BF4].

iv) A particular form of $x$-dependent problems arises in the theory of electrorheological fluids; we refer to $[\mathrm{R}, \mathrm{E}, \mathrm{ER}, \mathrm{AM}, \overline{\mathrm{BF}}$, $, \mathrm{BFZ}, \mathrm{DER}$, and the references quoted therein. Roughly speaking, the potential $f$ here is of the principal form $f(x, \varepsilon)=\left(1+|\varepsilon|^{2}\right)^{p(x) / 2}$ for a smooth function such that $1<p_{\infty} \leq p(x) \leq p_{0}<\infty$ with constants $p_{\infty}$ and $p_{0}$. The existence of a strong solution of (1.1) can be established by working in appropriate function spaces (see $[\mathrm{R}, \mathrm{E}]$ and $[\mathrm{ER}]$ ), and for the 2D-case the regularity of this solution follows from the observation that locally we have the bound $p \leq p(x) \leq \bar{q}$ with "local exponents" $p$ and $\bar{q}$ such that (1.5) is fulfilled together with $\bar{q}<p+2$.

\section{§2. Existence of A Strong SOlution}

Throughout this section we assume that all the hypotheses of the first part of Theorem 1.1 are satisfied. For vectors $u, v \in \mathbb{R}^{n}$, we let $u \otimes v=\left(u_{i} v_{j}\right)_{1 \leq i, j \leq n}$ and $u \odot v=$ $\frac{1}{2}(u \otimes v+v \otimes u)$.

We introduce the regularization $(0<\delta<1)$

$$
f_{\delta}(x, \varepsilon):=\delta\left(1+|\varepsilon|^{2}\right)^{\frac{q}{2}}+f(x, \varepsilon)
$$


with exponent $q$ satisfying (compare $[\mathrm{ABF}$, Remark 1.5])

$$
q>\bar{q}, \quad n<q<p\left(1+\frac{2}{n}\right)
$$

which is possible by our assumptions on the data. Let $u_{\delta}$ denote a solution in $\stackrel{\circ}{W}_{q}^{1}\left(\Omega ; \mathbb{R}^{n}\right) \cap$ Ker(div) of the problem

$$
\begin{array}{r}
\int_{\Omega} D_{\varepsilon} f_{\delta}(\cdot, \varepsilon(w)): \varepsilon(\varphi) \mathrm{d} x-\int_{\Omega} w \otimes w: \varepsilon(\varphi) \mathrm{d} x=\int_{\Omega} g \cdot \varphi \mathrm{d} x \\
\text { for all } \varphi \in C_{0}^{\infty}\left(\Omega ; \mathbb{R}^{n}\right), \operatorname{div} \varphi=0,
\end{array}
$$

whose existence follows from a familiar fixed point argument, which was applied for example in BF3, Appendix], and which can be used in our setting without changes. Choosing $\varphi=u_{\delta}$, we immediately get

$$
\sup _{0<\delta<1}\left\|u_{\delta}\right\|_{W_{p}^{1}\left(\Omega ; \mathbb{R}^{n}\right)}<\infty
$$

and we shall show that any weak $W_{p}^{1}$-cluster point $v$ of the sequence $\left\{u_{\delta}\right\}$ satisfies our claim. To this purpose, we follow the ideas of [BF1] and [ABF]: first, we establish some weak differentiability properties of the functions $u_{\delta}$ (see Lemma 2.1), which in turn enable us to prove a preliminary version of a Caccioppoli-type inequality (see Lemma 2.2). From this inequality we deduce some initial local higher integrability result for $\nabla u_{\delta}$ (uniformly in $\delta$; see Lemma 2.3), which gives an improved version of the first Caccioppolitype inequality (see Lemma 2.4). At the final step, we shall increase the exponent of the uniform local higher integrability (at least in the case where $n=3$ together with $p<2$; see Lemma 2.5). Putting all these ingredients together, we shall arrive at part i) of Theorem 1.1. We start with a lemma.

Lemma 2.1. We have the following initial regularity properties of $u_{\delta}$ :

i) $u_{\delta} \in W_{2, \text { loc }}^{2}\left(\Omega ; \mathbb{R}^{n}\right)$;

ii) $D_{\varepsilon} f_{\delta}\left(\cdot, \varepsilon\left(u_{\delta}\right)\right) \in W_{q /(q-1), \text { loc }}^{1}\left(\Omega ; \mathbb{S}^{n}\right)$ together with

$$
\begin{array}{r}
\partial_{k}\left\{D_{\varepsilon} f_{\delta}\left(\cdot, \varepsilon\left(u_{\delta}\right)\right)\right\}=D_{\varepsilon}^{2} f_{\delta}\left(\cdot, \varepsilon\left(u_{\delta}\right)\right)\left(\partial_{k} \varepsilon\left(u_{\delta}\right), \cdot\right)+\left(\partial_{k} D_{\varepsilon} f_{\delta}\right)\left(\cdot, \varepsilon\left(u_{\delta}\right)\right), \\
k=1, \ldots, n ;
\end{array}
$$

iii) $h_{\delta}:=\left(1+\left|\varepsilon\left(u_{\delta}\right)\right|^{2}\right)^{p / 4} \in W_{2, \text { loc }}^{1}(\Omega)$ together with

$$
\nabla h_{\delta}=\frac{p}{2}\left(1+\left|\varepsilon\left(u_{\delta}\right)\right|^{2}\right)^{\frac{p}{4}-1}\left|\varepsilon\left(u_{\delta}\right)\right| \nabla\left|\varepsilon\left(u_{\delta}\right)\right| .
$$

Proof. The idea of the proof is the same as in [BF1, Lemma 3.1] and [ABF, Lemma 2.2]; we only indicate the minor adjustments. We fix a ball $B_{R}=B_{R}\left(x_{0}\right) \Subset \Omega$ and choose $\eta \in C_{0}^{\infty}\left(B_{R}\right)$ such that $\left(0<r<r^{\prime}<R\right) \eta \equiv 1$ on $B_{r}, \eta \equiv 0$ outside of $B_{r^{\prime}}$, and $|\nabla \eta| \leq c /\left(r^{\prime}-r\right)$. Let $\Delta_{h}$ denote the difference quotient in the direction $e_{k}, k=1, \ldots, n$, and consider a function $\psi \in \stackrel{\circ}{W} \underset{q}{1}\left(B_{r^{\prime}} ; \mathbb{R}^{n}\right)$ such that

$$
\operatorname{div} \psi=\frac{1}{h} \nabla \eta^{2} \Delta_{h} u_{\delta}
$$

together with

$$
\|\nabla \psi\|_{L^{q}\left(B_{r^{\prime}}\right)} \leq c\left\|h^{-1} \nabla \eta^{2} \Delta_{h} u_{\delta}\right\|_{L^{q}\left(B_{r^{\prime}}\right)} .
$$

Note that such a function $\psi$ exists by [La, Pi] or [Ga, III, Theorem 3.2]. Then

$$
\varphi:=h^{-1} \eta^{2} \Delta_{h} u_{\delta}-\psi
$$


is admissible in $\left(1.1_{\delta}\right)$, which means that we get $(2.5)$ of $[\mathrm{ABF}]$ with $f_{\delta}\left(\cdot, \varepsilon\left(u_{\delta}\right)\right)$ replacing $f_{\delta}\left(\varepsilon\left(u_{\delta}\right)\right)$ on both sides of the equation. For the reader's convenience, we give some details. First, from $\left(1.1_{\delta}\right)$ it follows that

$$
\begin{aligned}
& \int_{B_{r^{\prime}}\left(x_{0}\right)} \Delta_{h}\left(D_{\varepsilon} f_{\delta}\left(\cdot, \varepsilon\left(u_{\delta}\right)\right)\right): \varepsilon(\varphi) \mathrm{d} x \\
& \quad=\int_{B_{r^{\prime}}\left(x_{0}\right)} \Delta_{h}\left(u_{\delta} \otimes u_{\delta}\right): \varepsilon(\varphi) \mathrm{d} x-\int_{B_{r^{\prime}}\left(x_{0}\right)} g \cdot \Delta_{-h} \varphi \mathrm{d} x
\end{aligned}
$$

and if, second, we insert $\varphi$ as chosen above, this equation gives

$$
\begin{aligned}
\int_{B_{r^{\prime}}\left(x_{0}\right)} & \Delta_{h}\left(D_{\varepsilon} f_{\delta}\left(\cdot, \varepsilon\left(u_{\delta}\right)\right)\right): \varepsilon\left(\Delta_{h} u_{\delta}\right) \eta^{2} \mathrm{~d} x \\
= & \int_{B_{r^{\prime}}\left(x_{0}\right)} \Delta_{h}\left(D_{\varepsilon} f_{\delta}\left(\cdot, \varepsilon\left(u_{\delta}\right)\right)\right):\left(h \varepsilon(\psi)-\nabla \eta^{2} \odot \Delta_{h} u_{\delta}\right) \mathrm{d} x \\
& +\int_{B_{r^{\prime}}\left(x_{0}\right)} \Delta_{h}\left(u_{\delta} \otimes u_{\delta}\right):\left(\eta^{2} \varepsilon\left(\Delta_{h} u_{\delta}\right)+\Delta_{h} u_{\delta} \odot \nabla \eta^{2}-h \varepsilon(\psi)\right) \mathrm{d} x \\
& -\int_{B_{r^{\prime}}\left(x_{0}\right)} g \cdot \Delta_{-h}\left(\eta^{2} \Delta_{h} u_{\delta}-h \psi\right) \mathrm{d} x,
\end{aligned}
$$

i.e., we obtain an appropriate version of (2.5) from $\underline{\mathrm{ABF}}$. We have

$$
\begin{aligned}
\Delta_{h}\left(D_{\varepsilon} f_{\delta}\left(\cdot, \varepsilon\left(u_{\delta}\right)\right)\right)(x) \\
=\frac{1}{h}\left[D_{\varepsilon} f_{\delta}\left(x+h e_{k}, \varepsilon\left(u_{\delta}\right)\left(x+h e_{k}\right)\right)-D_{\varepsilon} f_{\delta}\left(x+h e_{k}, \varepsilon\left(u_{\delta}\right)(x)\right)\right] \\
\quad+\frac{1}{h}\left[D_{\varepsilon} f_{\delta}\left(x+h e_{k}, \varepsilon\left(u_{\delta}\right)(x)\right)-D_{\varepsilon} f_{\delta}\left(x, \varepsilon\left(u_{\delta}\right)(x)\right)\right] \\
=\int_{0}^{1} D_{\varepsilon}^{2} f_{\delta}\left(x+h e_{k}, \varepsilon\left(u_{\delta}\right)(x)+\operatorname{th} \varepsilon\left(\Delta_{h} u_{\delta}\right)(x)\right) \mathrm{d} t\left(\varepsilon\left(\Delta_{h} u_{\delta}\right), \cdot\right) \\
\quad+\frac{1}{h}\left[D_{\varepsilon} f_{\delta}\left(x+h e_{k}, \varepsilon\left(u_{\delta}\right)(x)\right)-D_{\varepsilon} f_{\delta}\left(x, \varepsilon\left(u_{\delta}\right)(x)\right)\right]=: I+\mathbb{I} .
\end{aligned}
$$

Note that in II we may replace $f_{\delta}$ by $f$ since the regularizing $\delta$-part does not depend on $x$. If we introduce the parameter-dependent bilinear form

$$
\tilde{\mathcal{B}}_{x}:=\int_{0}^{1} D_{\varepsilon}^{2} f_{\delta}\left(x+h e_{k}, \varepsilon\left(u_{\delta}\right)(x)+t h \varepsilon\left(\Delta_{h} u_{\delta}\right)(x)\right) \mathrm{d} t
$$

then we obtain (2.6) of $\underline{\mathrm{ABF}}$ with $\mathcal{B}_{x}$ replaced by $\tilde{\mathcal{B}}_{x}$ and with some extra terms on the right-hand side, which originate from the new expression II. Moreover, on the left-hand side of the inequality mentioned above we may write

$$
\int_{B_{r^{\prime}}\left(x_{0}\right)} \tilde{\mathcal{B}}_{x}\left(\varepsilon\left(\Delta_{h} u_{\delta}\right), \varepsilon\left(\Delta_{h} u_{\delta}\right)\right) \eta^{2} \mathrm{~d} x
$$

which is helpful for absorbing terms. To be precise, exactly the same calculations as 
presented in $\mathrm{ABF}$ or $\mathrm{BF} 1$ imply the following starting inequality, replacing (2.6) of $\mathrm{ABF}$ :

$$
\begin{aligned}
\int_{B_{r^{\prime}}\left(x_{0}\right)} & \tilde{\mathcal{B}}_{x}\left(\varepsilon\left(\Delta_{h} u_{\delta}\right), \varepsilon\left(\Delta_{h} u_{\delta}\right)\right) \eta^{2} \mathrm{~d} x \\
\leq & \frac{1}{2} \int_{B_{r^{\prime}}\left(x_{0}\right)} \tilde{\mathcal{B}}_{x}\left(\varepsilon\left(\Delta_{h} u_{\delta}\right), \varepsilon\left(\Delta_{h} u_{\delta}\right)\right) \mathrm{d} x \\
+ & \frac{c}{\left(r^{\prime}-r\right)^{2}}\left[\int_{B_{r^{\prime}}\left(x_{0}\right)}\left|\Delta_{h} u_{\delta}\right|^{q} \mathrm{~d} x\right]^{\frac{2}{q}} \\
& \times\left[\int_{B_{r^{\prime}}\left(x_{0}\right)}\left(1+\left|\varepsilon\left(u_{\delta}\right)\right|^{2}+\left|h \varepsilon\left(\Delta_{h} u_{\delta}\right)\right|^{2}\right)^{\frac{q}{2}} \mathrm{~d} x\right]^{1-\frac{2}{q}} \\
+ & \left|\int_{B_{r^{\prime}}\left(x_{0}\right)} \Delta_{h}\left(u_{\delta} \otimes u_{\delta}\right):\left[\varepsilon\left(\Delta_{h} u_{\delta}\right) \eta^{2}+\Delta_{h} u_{\delta} \odot \nabla \eta^{2}-h \varepsilon(\psi)\right] \mathrm{d} x\right| \\
+ & \left|\int_{B_{r^{\prime}}\left(x_{0}\right)} g \cdot \Delta_{-h}\left(\eta^{2} \Delta_{h} u_{\delta}-h \psi\right) \mathrm{d} x\right|+\left|\int_{B_{r^{\prime}}\left(x_{0}\right)} I I: \varepsilon\left(\eta^{2} \Delta_{h} u_{\delta}-h \psi\right) \mathrm{d} x\right| .
\end{aligned}
$$

Next, we use the growth condition (1.3) in order to estimate the last term on the righthand side:

$$
\begin{aligned}
& \int_{B_{r^{\prime}}\left(x_{0}\right)}|I I|\left|\varepsilon\left(\eta^{2} \Delta_{h} u_{\delta}-h \psi\right)\right| \mathrm{d} x \\
& \leq c\left[\int_{B_{r^{\prime}}\left(x_{0}\right)} \Gamma_{\delta}^{\frac{\bar{q}-1}{2}} \eta^{2}\left|\varepsilon\left(\Delta_{h} u_{\delta}\right)\right| \mathrm{d} x\right. \\
& \left.\quad+\int_{B_{r^{\prime}}\left(x_{0}\right)} \Gamma_{\delta}^{\frac{\bar{q}-1}{2}} \eta\left|\nabla \eta \| \Delta_{h} u_{\delta}\right| \mathrm{d} x+\int_{B_{r^{\prime}}\left(x_{0}\right)} \Gamma_{\delta}^{\frac{\bar{q}-1}{2}}|h||\varepsilon(\psi)| \mathrm{d} x\right] \\
& =: c\left[T_{1}+T_{2}+T_{3}\right],
\end{aligned}
$$

$\Gamma_{\delta}:=1+\left|\varepsilon\left(u_{\delta}\right)\right|^{2}$. Let $\sigma \in \mathbb{S}^{n}$. Then, using (1.2) and the definition of $f_{\delta}$, we have

$$
\begin{aligned}
\tilde{\mathcal{B}}_{x}(\sigma, \sigma) & \geq \lambda(\delta) \int_{0}^{1}\left(1+\left|\varepsilon\left(u_{\delta}\right)(x)+\operatorname{th} \varepsilon\left(\Delta_{h} u_{\delta}\right)(x)\right|^{2}\right)^{\frac{q-2}{2}} \mathrm{~d} t|\sigma|^{2} \\
& \geq c(\delta) \Gamma_{\delta}^{\frac{q-2}{2}}|\sigma|^{2},
\end{aligned}
$$

where the last inequality follows from well-known estimates valid because $q>2$. This gives

$$
\begin{aligned}
T_{1} & \leq c(\delta) \int_{B_{r^{\prime}}\left(x_{0}\right)} \eta^{2} \Gamma_{\delta}^{\frac{\bar{q}-1}{2}} \Gamma_{\delta}^{\frac{2-q}{4}}\left[\tilde{\mathcal{B}}_{x}\left(\varepsilon\left(\Delta_{h} u_{\delta}\right), \varepsilon\left(\Delta_{h} u_{\delta}\right)\right)\right]^{\frac{1}{2}} \mathrm{~d} x \\
& \leq \rho \int_{B_{r^{\prime}}\left(x_{0}\right)} \eta^{2} \tilde{\mathcal{B}}_{x}\left(\varepsilon\left(\Delta_{h} u_{\delta}\right), \varepsilon\left(\Delta_{h} u_{\delta}\right)\right) \mathrm{d} x+c(\delta, \rho) \int_{B_{r^{\prime}}\left(x_{0}\right)} \eta^{2} \Gamma_{\delta}^{\bar{q}-1+\frac{2-q}{2}} \mathrm{~d} x,
\end{aligned}
$$

and for $\rho \ll 1$ the first term can be absorbed into the left-hand side of the starting inequality, whereas the second integral is dominated by $\int_{B_{r^{\prime}}\left(x_{0}\right)} \Gamma_{\delta}^{q / 2} \mathrm{~d} x$. Obviously,

$$
\begin{aligned}
T_{2} & \leq c\left(r^{\prime}-r\right)^{-1}\left[\int_{B_{r^{\prime}}\left(x_{0}\right)}\left|\Delta_{h} u_{\delta}\right|^{q} \mathrm{~d} x+\int_{B_{r^{\prime}}\left(x_{0}\right)} \Gamma_{\delta}^{\frac{\bar{q}-1}{2} \frac{q}{q-1}} \mathrm{~d} x\right] \\
& \leq c\left(r^{\prime}-r\right)^{-1} \int_{B_{R+h}\left(x_{0}\right)} \tilde{\Gamma}_{\delta}^{\frac{q}{2}} \mathrm{~d} x, \quad \tilde{\Gamma}_{\delta}:=1+\left|\nabla u_{\delta}\right|^{2},
\end{aligned}
$$

which is a consequence of $\frac{\bar{q}-1}{2} \frac{q}{q-1} \leq \frac{q}{2}$. 
For $T_{3}$ we get the same estimate, recalling the bound for $\int_{B_{r^{\prime}}\left(x_{0}\right)}|\nabla \psi|^{q} \mathrm{~d} x$. This finishes the discussion of the last term on the right-hand side of the starting inequality; all other quantities already occur in (2.6) of $\mathrm{ABF}$ and can be handled as demonstrated there. This means that, finally, inequality (2.10) of $\mathrm{ABF}$ is established with some extra term of the form $c\left(r^{\prime}-r\right)^{-2} \int_{B_{R+h}\left(x_{0}\right)} \tilde{\Gamma}_{\delta}^{q / 2} \mathrm{~d} x$ on the right-hand side; thus a substitute for (3.9) of BF1 follows (with $s$ replaced by $q$ and $\mathcal{B}_{x}$ replaced by $\tilde{\mathcal{B}}_{x}$ ) and part i) of Lemma 2.1 is a consequence of the fact that $q \geq 2$. The remaining statements of Lemma 2.1 can now be adjusted along the lines of [BF1, pp. 373 and 374].

Lemma 2.2. Consider a ball $B_{R}=B_{R}\left(x_{0}\right) \Subset \Omega$ and choose radii $0<r<r^{\prime}<R$. Then there exists a local constant $c\left(r, r^{\prime}\right)=c\left(r^{\prime}-r\right)^{-\beta}, \beta>0$, such that for any ball $B_{r^{\prime}}(\bar{x}) \Subset B_{R}$ and for any $\eta \in C_{0}^{\infty}\left(B_{r^{\prime}}(\bar{x})\right)$ with $0 \leq \eta \leq 1, \eta \equiv 1$ on $B_{r}(\bar{x})$, and $|\nabla \eta| \leq c\left(r^{\prime}-r\right)^{-1}$, the following estimate is true:

$$
\begin{aligned}
\int_{B_{r^{\prime}}(\bar{x})} \eta^{2} \Gamma_{\delta}^{\frac{p-2}{2}}\left|\nabla \varepsilon\left(u_{\delta}\right)\right|^{2} \mathrm{~d} x & \leq c\left(r, r^{\prime}\right)[1 \\
\leq & \int_{B_{r^{\prime}}(\bar{x})} \Gamma_{\delta}^{\frac{2 \bar{q}-p}{2}} \mathrm{~d} x+\int_{B_{r^{\prime}}(\bar{x})} \Gamma_{\delta}^{\frac{q}{2}} \mathrm{~d} x \\
& \left.+\int_{B_{r^{\prime}}(\bar{x})}\left|u_{\delta}\right|\left|\nabla u_{\delta}\right|^{2} \mathrm{~d} x+\left|\int_{B_{r^{\prime}}(\bar{x})} \partial_{k} u_{\delta}^{j} \partial_{j} u_{\delta}^{i} \partial_{k} u_{\delta}^{i} \eta^{2} \mathrm{~d} x\right|\right],
\end{aligned}
$$

where the last integral on the right-hand side vanishes if $n=2$. Again we have set $\Gamma_{\delta}:=1+\left|\varepsilon\left(u_{\delta}\right)\right|^{2}$.

Remark 2.1. Note that by (1.5),

$$
2 \bar{q}-p<2 p\left(1+\frac{1}{n}\right)-p=p\left(1+\frac{2}{n}\right) ;
$$

hence we may choose $q$ such that, in addition to (2.1),

$$
2 \bar{q}-p<q .
$$

Then the claim of Lemma 2.2 exactly takes the form of [ABF, Lemma 3.1], which means that we can drop the integral $\int_{B_{r^{\prime}}(\bar{x})} \Gamma_{\delta}^{(2 \bar{q}-p) / 2} \mathrm{~d} x$ in the inequality stated in Lemma 2.2 ,

Proof of Lemma 2.2. Using the notation in [ABF, proof of Lemma 3.1], with exactly the same calculations we obtain inequality (3.5) in that paper; i.e., we have $\left(\sigma_{\delta}:=\right.$ $\left.D_{\varepsilon} f_{\delta}\left(\cdot, \varepsilon\left(u_{\delta}\right)\right)\right)$

$$
\begin{aligned}
& \int_{B_{r^{\prime}}(\bar{x})} \eta^{2} \partial_{k} \sigma_{\delta}: \partial_{k} \varepsilon\left(u_{\delta}\right) \mathrm{d} x \\
& \leq-2 \int_{B_{r^{\prime}}(\bar{x})} \eta \partial_{k} \sigma_{\delta}:\left(\nabla \eta \odot \partial_{k} u_{\delta}\right) \mathrm{d} x \\
& \quad+\int_{B_{r^{\prime}}(\bar{x})} \partial_{k}\left(u_{\delta} \otimes u_{\delta}\right): \varepsilon\left(\eta^{2} \partial_{k} u_{\delta}\right) \mathrm{d} x \\
& \quad-\int_{B_{r^{\prime}}(\bar{x})} g \partial_{k}\left(\eta^{2} \partial_{k} u_{\delta}\right) \mathrm{d} x-2 \int_{B_{r^{\prime}}(\bar{x})} \eta \partial_{k} \pi_{\delta} \mathbf{1}:\left(\nabla \eta \odot \partial_{k} u_{\delta}\right) \mathrm{d} x,
\end{aligned}
$$

where $\pi_{\delta}$ is the pressure function defined as in [Ga, p. 180, Lemma 1.1]: to be precise, the growth of $f$ implies $\sigma_{\delta} \in L^{q /(q-1)}\left(B_{R}\left(x_{0}\right) ; \mathbb{S}^{n}\right),\left|u_{\delta} \otimes u_{\delta}\right| \in L^{q /(q-1)}\left(B_{R}\left(x_{0}\right)\right)$ is immediate, and we recall that $g \in L^{\infty}\left(\Omega ; \mathbb{R}^{n}\right)$. As a result,

$$
\Phi: \stackrel{\circ}{W_{q}^{1}}\left(B_{R}\left(x_{0}\right) ; \mathbb{R}^{n}\right) \ni \varphi \mapsto \int_{B_{R}\left(x_{0}\right)}\left[\sigma_{\delta}-u_{\delta} \otimes u_{\delta}\right]: \varepsilon(\varphi) \mathrm{d} x-\int_{B_{R}\left(x_{0}\right)} g \cdot \varphi \mathrm{d} x
$$


belongs to the dual space $\stackrel{\circ}{W}_{q}^{1}\left(B_{R}\left(x_{0}\right) ; \mathbb{R}^{n}\right)^{*}$ and satisfies $\Phi(\varphi)=0$ if $\operatorname{div} \varphi=0$ (see $\left(1.1_{\delta}\right)$ ). Therefore, we find a function $\pi_{\delta} \in L^{q /(q-1)}\left(B_{R}\left(x_{0}\right)\right)$ such that $\int_{B_{R}\left(x_{0}\right)} \pi_{\delta} \mathrm{d} x=0$ and $\Phi(\varphi)=\int_{B_{R}\left(x_{0}\right)} \pi_{\delta} \operatorname{div} \varphi \mathrm{d} x$ for all $\varphi \in \stackrel{\circ}{W_{q}^{1}}\left(B_{R}\left(x_{0}\right) ; \mathbb{R}^{n}\right)$. To estimate the left-hand side of (2.2), we use (compare the formula in ii) of Lemma 2.1)

$$
\begin{aligned}
\partial_{k} \sigma_{\delta}: \varepsilon\left(\partial_{k} u_{\delta}\right) & =\underbrace{D_{\varepsilon}^{2} f_{\delta}\left(\cdot, \varepsilon\left(u_{\delta}\right)\right)\left(\partial_{k} \varepsilon\left(u_{\delta}\right), \partial_{k} \varepsilon\left(u_{\delta}\right)\right)}_{=: H_{\delta}^{2}}+\left(\partial_{k} D_{\varepsilon} f_{\delta}\right)\left(\cdot, \varepsilon\left(u_{\delta}\right)\right): \varepsilon\left(\partial_{k} u_{\delta}\right) \\
& \geq H_{\delta}^{2}-c \Gamma_{\delta}^{\bar{q}-1}\left|\nabla \varepsilon\left(u_{\delta}\right)\right|,
\end{aligned}
$$

where the inequality follows from (1.3). Moreover, we observe that

$$
\Gamma_{\delta}^{\frac{\bar{q}-1}{2}}\left|\nabla \varepsilon\left(u_{\delta}\right)\right| \leq \tau \Gamma_{\delta}^{\frac{p-2}{2}}\left|\nabla \varepsilon\left(u_{\delta}\right)\right|^{2}+c(\tau) \Gamma_{\delta}^{\frac{2 \bar{q}-p}{2}}
$$

$(0<\tau<1)$. Thus, after an appropriate choice of $\tau$ (recall the ellipticity estimate (1.2) ), we get the bound

$$
\partial_{k} \sigma_{\delta}: \varepsilon\left(\partial_{k} u_{\delta}\right) \geq \frac{1}{2} H_{\delta}^{2}-c \Gamma_{\delta}^{\frac{2 \bar{q}-p}{2}},
$$

the constant $c$ being uniform with respect to $\delta$. For estimating the first integral on the right-hand side of (2.2), we observe that

$$
\begin{aligned}
\left|\nabla \sigma_{\delta}\right|^{2} & =\partial_{k} \sigma_{\delta}: \partial_{k} \sigma_{\delta} \\
& =D_{\varepsilon}^{2} f_{\delta}\left(\cdot, \varepsilon\left(u_{\delta}\right)\right)\left(\partial_{k} \varepsilon\left(u_{\delta}\right), \partial_{k} \sigma_{\delta}\right)+\left(\partial_{k} D_{\varepsilon} f_{\delta}\right)\left(\cdot, \varepsilon\left(u_{\delta}\right)\right): \partial_{k} \sigma_{\delta} \\
& \leq H_{\delta} D_{\varepsilon}^{2} f_{\delta}\left(\cdot, \varepsilon\left(u_{\delta}\right)\right)\left(\partial_{k} \sigma_{\delta}, \partial_{k} \sigma_{\delta}\right)^{\frac{1}{2}}+c \Gamma_{\delta}^{\frac{\bar{q}-1}{2}}\left|\nabla \sigma_{\delta}\right|,
\end{aligned}
$$

which gives

$$
\left|\nabla \sigma_{\delta}\right| \leq c H_{\delta} \Gamma_{\delta}^{\frac{q-2}{4}}+c \Gamma_{\delta}^{\frac{\bar{q}-1}{2}}
$$

Now (2.4) implies that

$$
\begin{aligned}
& 2 \int_{B_{r^{\prime}}(\bar{x})} \eta\left|\nabla \sigma_{\delta}\right||\nabla \eta|\left|\nabla u_{\delta}\right| \mathrm{d} x \\
& \quad \leq c \int_{B_{r^{\prime}}(\bar{x})} \eta H_{\delta} \Gamma_{\delta}^{\frac{q-2}{4}}|\nabla \eta|\left|\nabla u_{\delta}\right| \mathrm{d} x+c \int_{B_{r^{\prime}}(\bar{x})} \eta|\nabla \eta|\left|\nabla u_{\delta}\right| \Gamma_{\delta}^{\frac{\bar{q}-1}{2}} \mathrm{~d} x \\
& \leq \tau \int_{B_{r^{\prime}(\bar{x})}} \eta^{2} H_{\delta}^{2} \mathrm{~d} x+c(\tau) \int_{B_{r^{\prime}}(\bar{x})}|\nabla \eta|^{2} \Gamma_{\delta}^{\frac{q-2}{2}}\left|\nabla u_{\delta}\right|^{2} \mathrm{~d} x \\
& \quad+c \int_{B_{r^{\prime}}(\bar{x})} \eta|\nabla \eta|\left|\nabla u_{\delta}\right| \Gamma_{\delta}^{\frac{\bar{q}-1}{2}} \mathrm{~d} x,
\end{aligned}
$$

and the first two terms on the right-hand side can be handled in a standard way (see the calculations after (3.6) in $[\mathrm{ABF}$. showing how to treat the second item). The last integral can be treated like the second, which leads to

$$
\int_{B_{r^{\prime}}(\bar{x})} \eta|\nabla \eta|\left|\nabla u_{\delta}\right| \Gamma_{\delta}^{\frac{\bar{q}-1}{2}} \mathrm{~d} x \leq c\left(r, r^{\prime}\right)\left[1+\int_{B_{r^{\prime}}(\bar{x})} \Gamma_{\delta}^{\frac{q}{2}} \mathrm{~d} x\right],
$$

where we have also used the assumption that $\bar{q}<q$.

The second and the third integral on the right-hand side of (2.2) can be estimated exactly as in $\overline{\mathrm{ABF}}$, and for the pressure integral we observe the equation

$$
g+\operatorname{div}\left(\sigma_{\delta}-u_{\delta} \otimes u_{\delta}\right)=\nabla \pi_{\delta} ;
$$


thus,

$$
\begin{aligned}
& \left|-2 \int_{B_{r^{\prime}}(\bar{x})} \eta \partial_{k} \pi_{\delta} \mathbf{1}:\left(\nabla \eta \odot \partial_{k} u_{\delta}\right) \mathrm{d} x\right| \\
& \leq c\left[\int_{B_{r^{\prime}}(\bar{x})} \eta|\nabla \eta|\left|\nabla \sigma_{\delta}\right|\left|\nabla u_{\delta}\right| \mathrm{d} x\right. \\
& \left.\quad+\int_{B_{r^{\prime}}(\bar{x})} \eta|\nabla \eta|\left|\nabla\left(u_{\delta} \otimes u_{\delta}\right)\right|\left|\nabla u_{\delta}\right| \mathrm{d} x+\int_{B_{r^{\prime}}(\bar{x})} \eta|\nabla \eta||g|\left|\nabla u_{\delta}\right| \mathrm{d} x\right] \\
& =: c\left[T_{1}+T_{2}+T_{3}\right] .
\end{aligned}
$$

The integrals $T_{2}, T_{3}$ already occurred, and $T_{1}$ was discussed after inequality (2.4). Now, the rest of the proof is the same as in $\underline{\mathrm{ABF}}$.

Lemma 2.3. For any subdomain $\Omega^{\prime} \Subset \Omega$ there is a constant $c\left(\Omega^{\prime}\right)$ independent of $\delta$ such that

$$
\int_{\Omega^{\prime}}\left|\nabla u_{\delta}\right|^{p^{*}} \mathrm{~d} x \leq c\left(\Omega^{\prime}\right)
$$

where

$$
p^{*}= \begin{cases}\frac{n p}{n-p} & \text { if } p<n, \\ \text { any number } & \text { if } p \geq n .\end{cases}
$$

Remark 2.2. Our assumptions (1.4) and (2.1) in particular imply that $p^{*}>q$, which follows for $p<n$ from the fact that $p>2 n /(n+2)$. In case $p \geq n$ we merely choose $p^{*}>q$.

Proof of Lemma 2.3. In view of Remark 2.1, the proof of Lemma 2.3 is a verbatim repetition of $\underline{\mathrm{ABF}}$, proof of Lemma 3.2].

As in $\overline{\mathrm{ABF}}$, we use Lemma 2.3 to improve the Caccioppoli-type inequality stated in Lemma 2.2.

Lemma 2.4. We use the notation of Lemma 2.2, There exists an exponent $\gamma>0$ and uniform local constants $c_{1}, c_{2}, c_{3}>0$ such that for any matrix $Q \in \mathbb{R}^{n \times n}$ we have

$$
\begin{aligned}
& \int_{B_{r^{\prime}}(\bar{x})} \Gamma_{\delta}^{\frac{p-2}{2}}\left|\nabla \varepsilon\left(u_{\delta}\right)\right|^{2} \mathrm{~d} x \\
& \quad \leq c_{1} \int_{B_{r^{\prime}}(\bar{x})} \Gamma_{\delta}^{\frac{2 \bar{q}-p}{2}} \mathrm{~d} x+\frac{c_{2}}{\left(r^{\prime}-r\right)^{2}} \int_{B_{r^{\prime}}(\bar{x})} \Gamma_{\delta}^{\frac{q-2}{2}}\left|\nabla u_{\delta}-Q\right|^{2} \mathrm{~d} x+c_{3}\left(r^{\prime}\right)^{\gamma} .
\end{aligned}
$$

Proof. If we replace $u_{\delta}$ by $u_{\delta}-Q x$ in the test function used in the proof of Lemma 3.1 of $\mathrm{ABF}$, then we obtain a version of inequality (2.2) where on the right-hand side the quantity $u_{\delta}-Q x$ occurs in place of $u_{\delta}$ at appropriate positions. Using the estimates stated after (2.2), taking also care of the uniform local boundedness of $u_{\delta}$ (which is a consequence of Lemma 2.3), and using again the equation $g+\operatorname{div}\left(\sigma_{\delta}-u_{\delta} \otimes u_{\delta}\right)=\nabla \pi_{\delta}$ 
for handling the pressure term, we arrive at the following result (compare with (3.15) of (ABF]):

$$
\begin{aligned}
\int_{B_{r^{\prime}}(\bar{x})} & \eta^{2} \Gamma_{\delta}^{\frac{p-2}{2}}\left|\nabla \varepsilon\left(u_{\delta}\right)\right|^{2} \mathrm{~d} x \\
\leq c & {\left[\int_{B_{r^{\prime}(\bar{x})}}|\nabla \eta|^{2} \Gamma_{\delta}^{\frac{q-2}{2}}\left|\nabla u_{\delta}-Q\right|^{2} \mathrm{~d} x+\int_{B_{r^{\prime}}(\bar{x})}|\nabla \eta| \eta \Gamma_{\delta}^{\frac{\bar{q}-1}{2}}\left|\nabla u_{\delta}-Q\right| \mathrm{d} x\right.} \\
& +\int_{B_{r^{\prime}}(\bar{x})} \Gamma_{\delta}^{\frac{2 \bar{q}-p}{2}} \mathrm{~d} x+\int_{B_{r^{\prime}}(\bar{x})} \eta|\nabla \eta|\left|\nabla u_{\delta}-Q\right|\left|\nabla u_{\delta}\right| \mathrm{d} x \\
& \left.+\left|\int_{B_{r^{\prime}}(\bar{x})} \partial_{k}\left(u_{\delta} \otimes u_{\delta}\right): \varepsilon\left(\eta \partial_{k}\left(u_{\delta}-Q x\right)\right) \mathrm{d} x\right|+\left|\int_{B_{r^{\prime}}(\bar{x})} g \partial_{k}\left(\eta^{2} \partial_{k}\left(u_{\delta}-Q x\right)\right) \mathrm{d} x\right|\right] \\
=: & c \sum_{i=1}^{6} T_{i} .
\end{aligned}
$$

The quantities $T_{1}, T_{5}$, and $T_{6}$ already occurred on the right-hand side of (3.15) in $\mathrm{ABF}$ and can be treated as demonstrated there. The term $T_{3}$ occurs on the right-hand side of the desired inequality, and clearly

$$
\begin{aligned}
T_{2} & \leq c \int_{B_{r^{\prime}}(\bar{x})}|\nabla \eta|^{2}\left|\nabla u_{\delta}-Q\right|^{2} \Gamma_{\delta}^{\frac{\bar{q}-2}{2}} \mathrm{~d} x+c \int_{B_{r^{\prime}}(\bar{x})} \Gamma_{\delta}^{\frac{\bar{q}}{2}} \mathrm{~d} x \\
& \leq c \int_{B_{r^{\prime}}(\bar{x})}|\nabla \eta|^{2}\left|\nabla u_{\delta}-Q\right|^{2} \Gamma_{\delta}^{\frac{q-2}{2}} \mathrm{~d} x+c \int_{B_{r^{\prime}}(\bar{x})} \Gamma_{\delta}^{\frac{2 \bar{q}-p}{2}} \mathrm{~d} x .
\end{aligned}
$$

For $T_{4}$ we get

$$
\begin{aligned}
T_{4} & \leq c\left(r^{\prime}-r\right)^{-2} \int_{B_{r^{\prime}}(\bar{x})}\left|\nabla u_{\delta}-Q\right|^{2} \mathrm{~d} x+\int_{B_{r^{\prime}}(\bar{x})}\left|\nabla u_{\delta}\right|^{2} \mathrm{~d} x \\
& \leq c\left[\left(r^{\prime}-r\right)^{-2} \int_{B_{r^{\prime}}(\bar{x})} \Gamma_{\delta}^{\frac{q-2}{2}}\left|\nabla u_{\delta}-Q\right|^{2} \mathrm{~d} x+\left(r^{\prime}\right)^{\gamma}\right],
\end{aligned}
$$

which follows from Lemma 2.3 This completes the proof of Lemma 2.4 .

Finally, we use the foregoing results to extend Theorem 1.2 of $\mathrm{ABF}$ to the situation studied here.

Lemma 2.5. The functions $u_{\delta}$ are of class $W_{\tilde{q}, \text { loc }}^{1}\left(\Omega ; \mathbb{R}^{n}\right)$ uniformly with respect to $\delta$, where $\tilde{q}=3 p$ if $n=3$, and where we may choose any finite number $\tilde{q}$ if $n=2$.

Remark 2.3. Note that, for example, in the 3D-case we have $3 p>p^{*}$ provided that $p<2$. Thus, Lemma 2.5 is an improvement of Lemma 2.3.

Proof of Lemma 2.5. We follow [BF1, proof of Lemma 4.4], and consider the case where $n=3$; the case of $n=2$ is left to the reader. From (2.1) we deduce that $p<q<\tilde{q}$; therefore, there exists $\theta \in(0,1)$ such that

$$
\frac{1}{q}=\frac{\theta}{p}+\frac{1-\theta}{\tilde{q}}
$$

Note that (2.5) and (2.1) imply that

$$
\frac{q}{p}(1-\theta)<1 .
$$

We fix a ball $B_{2 R}\left(x_{0}\right) \Subset \Omega$ and consider $0<r<r^{\prime}$ and $\bar{x}$ such that $B_{r^{\prime}}(\bar{x}) \Subset B_{R}=$ $B_{R}\left(x_{0}\right)$. Finally, let $0 \leq \eta \in C_{0}^{\infty}\left(B_{2 R}\right)$ be such that $\eta \equiv 1$ on $B_{r}(\bar{x})$. From Sobolev's 
inequality we deduce that

$$
\begin{aligned}
\int_{B_{r}(\bar{x})} \Gamma_{\delta}^{\frac{3}{2} p} \mathrm{~d} x & \leq \int_{B_{2 R}}\left(\eta h_{\delta}\right)^{6} \mathrm{~d} x \\
& \leq c\left[\int_{B_{2 R}}\left|\nabla\left(\eta h_{\delta}\right)\right|^{2} \mathrm{~d} x\right]^{3} \\
& \leq c\left[\int_{B_{2 R}}|\nabla \eta|^{2} h_{\delta}^{2} \mathrm{~d} x+\int_{B_{2 R}} \eta^{2}\left|\nabla h_{\delta}\right|^{2} \mathrm{~d} x\right]^{3}=: c\left[T_{1}+T_{2}\right]^{3},
\end{aligned}
$$

where $h_{\delta}$ was defined in Lemma 2.1. Clearly,

$$
T_{1} \leq c\|\nabla \eta\|_{L^{\infty}\left(B_{2 R}\right)}^{2} \int_{B_{2 R}} \Gamma_{\delta}^{\frac{p}{2}} \mathrm{~d} x
$$

and

$$
T_{2} \leq c \int_{B_{2 R}} \eta^{2} D_{\varepsilon}^{2} f_{\delta}\left(\cdot, \varepsilon\left(u_{\delta}\right)\right)\left(\partial_{k} \varepsilon\left(u_{\delta}\right), \partial_{k} \varepsilon\left(u_{\delta}\right)\right) \mathrm{d} x
$$

by the ellipticity estimate (1.2). Note again that all constants are uniform with respect to $\delta$. Assume now that $\eta \equiv 0$ outside of $B_{\left(r+r^{\prime}\right) / 2}(\bar{x})$ and that $|\nabla \eta| \leq c /\left(r^{\prime}-r\right)$. Then, obviously,

$$
\begin{aligned}
T_{2} & \leq c \int_{B_{\left(r+r^{\prime}\right) / 2}(\bar{x})} D_{\varepsilon}^{2} f_{\delta}\left(\cdot, \varepsilon\left(u_{\delta}\right)\right)\left(\partial_{k} \varepsilon\left(u_{\delta}\right), \partial_{k} \varepsilon\left(u_{\delta}\right)\right) \mathrm{d} x \\
& \leq c\left[\int_{B_{r^{\prime}}(\bar{x})} \Gamma_{\delta}^{\frac{2 \bar{q}-p}{2}} \mathrm{~d} x+\left(r^{\prime}-r\right)^{-2} \int_{B_{r^{\prime}}(\bar{x})} \Gamma_{\delta}^{\frac{q-2}{2}}\left|\nabla u_{\delta}-Q\right|^{2} \mathrm{~d} x+\left(r^{\prime}\right)^{\gamma}\right],
\end{aligned}
$$

where we have used Lemma 2.4. As outlined in the proof of Corollary 4.1 in [BF1], it is possible to choose the matrix $Q$ in such a way that

$$
\int_{B_{r^{\prime}}(\bar{x})} \Gamma_{\delta}^{\frac{q-2}{2}}\left|\nabla u_{\delta}-Q\right|^{2} \mathrm{~d} x \leq c \int_{B_{r^{\prime}}(\bar{x})} \Gamma_{\delta}^{\frac{q}{2}} \mathrm{~d} x
$$

Thus, we have shown that

$$
\begin{aligned}
{\left[\int_{B_{r}(\bar{x})} \Gamma_{\delta}^{\frac{3}{2} p} \mathrm{~d} x\right]^{\frac{1}{3}} \leq } & c\left(r^{\prime}-r\right)^{-2}\left[\int_{B_{2 R}} \Gamma_{\delta}^{\frac{p}{2}} \mathrm{~d} x+\int_{B_{r^{\prime}}(\bar{x})} \Gamma_{\delta}^{\frac{q}{2}} \mathrm{~d} x\right] \\
& +c\left[\left(r^{\prime}\right)^{\gamma}+\int_{B_{r^{\prime}}(\bar{x})} \Gamma_{\delta}^{\frac{2 \bar{q}-p}{2}} \mathrm{~d} x\right] .
\end{aligned}
$$

Recalling Remark 2.1 and Remark 2.2, we see that (2.7) differs from inequality (4.17) in BF1 merely by a uniform local constant, and the lemma follows precisely as outlined in [BF1, via an interpolation argument leading to (4.18) of [BF1] with exponent $\theta$ chosen in accordance with (2.5). Since we have (2.6), the arguments after (4.18) can be copied without changes.

After these preparations, we show that, actually, any weak $W_{p}^{1}$-cluster point of the sequence $\left\{u_{\delta}\right\}$ (whose existence follows from $\sup _{0<\delta<1}\left\|u_{\delta}\right\|_{W_{p}^{1}\left(\Omega ; \mathbb{R}^{n}\right)}<\infty$ ) is a strong solution of (1.1). Let $u_{\delta} \neg: u$ in $W_{p}^{1}\left(\Omega ; \mathbb{R}^{n}\right)$ as $\delta \rightarrow 0$. As in [ABF, $\S 4$ ], from Lemma 2.5 we deduce that

$$
D_{\varepsilon} f_{\delta}\left(\cdot, \varepsilon\left(u_{\delta}\right)\right) \in W_{q /(q-1), \mathrm{loc}}^{1}\left(\Omega ; \mathbb{S}^{n}\right)
$$

uniformly with respect to $\delta$, and as in $[\mathrm{ABF}$ we can show that

$$
D_{\varepsilon} f_{\delta}\left(\cdot, \varepsilon\left(u_{\delta}\right)\right) \stackrel{\delta \rightarrow 0}{\longrightarrow} D_{\varepsilon} f(\cdot, \varepsilon(u))
$$


strongly in $L_{\text {loc }}^{q /(q-1)}\left(\Omega ; \mathbb{S}^{n}\right)$ and a.e. This implies that

$$
\int_{\Omega} D_{\varepsilon} f(\cdot, \varepsilon(u)): \varepsilon(\varphi) \mathrm{d} x=\int_{\Omega} u \otimes u: \varepsilon(\varphi) \mathrm{d} x+\int_{\Omega} g \cdot \varphi \mathrm{d} x
$$

for any $\varphi \in C_{0}^{\infty}\left(\Omega ; \mathbb{R}^{n}\right)$ with $\operatorname{div} \varphi=0$. Combining Lemma 2.3 and Lemma 2.4 , we find

$$
\int_{\Omega^{\prime}} \Gamma_{\delta}^{\frac{p-2}{2}}\left|\nabla \varepsilon\left(u_{\delta}\right)\right|^{2} \mathrm{~d} x \leq c\left(\Omega^{\prime}\right)<\infty
$$

for any $\Omega^{\prime} \Subset \Omega$ uniformly with respect to $\delta$. If $p \geq 2$, then obviously we get

$$
\left\|u_{\delta}\right\|_{W_{2}^{2}\left(\Omega^{\prime} ; \mathbb{R}^{n}\right)} \leq c\left(\Omega^{\prime}\right)<\infty .
$$

If $p<2$, then Lemma 2.5 together with Young's inequality implies the bound

$$
\left\|u_{\delta}\right\|_{W_{\alpha}^{2}\left(\Omega^{\prime} ; \mathbb{R}^{n}\right)} \leq c\left(\Omega^{\prime}\right)<\infty
$$

for any exponent $\alpha<3 p /(p+1)$ in the 3D-case and for any number $\alpha<2$ if $n=2$. This shows that $u \in W_{t, \text { loc }}^{2}\left(\Omega ; \mathbb{R}^{n}\right)$ for a suitable exponent $t$; hence $u$ is a strong solution of equation (1.1). This proves i) of Theorem 1.1 .

\section{§3. Planar Flows}

In this section we give the proof of statement ii) of Theorem 1.1 assuming from now on that all the hypotheses of that theorem are valid.

We recall the following lemma on the higher integrability of functions, which was proved in BFZ, Lemma 1.2].

Lemma 3.1. Let $d>1, \beta>0$ be two constants. With a slight abuse of notation, let $f, g, h$ denote any nonnegative functions in $\Omega \subset \mathbb{R}^{n}$ satisfying $f \in L_{\text {loc }}^{d}(\Omega), \exp \left(\beta g^{d}\right) \in$ $L_{\mathrm{loc}}^{1}(\Omega)$, and $h \in L_{\mathrm{loc}}^{d}(\Omega)$. Suppose that there is a constant $C>0$ such that

$$
\left(f_{B} f^{d} d x\right)^{\frac{1}{d}} \leq C f_{2 B} f g \mathrm{~d} x+C\left(f_{2 B} h^{d} \mathrm{~d} x\right)^{\frac{1}{d}}
$$

for all balls $B=B_{r}(x)$ with $2 B=B_{2 r}(x) \Subset \Omega$. Then there is a real number $c_{0}=$ $c_{0}(n, d, C)>0$ such that if $h^{d} \log ^{c_{0} \beta}(e+h) \in L_{\mathrm{loc}}^{1}(\Omega)$, then the same is true for $f$. Moreover, for all balls $B$ as above we have

$$
\begin{aligned}
f_{B} & f^{d} \log ^{c_{0} \beta}\left(e+\frac{f}{\|f\|_{d, 2 B}}\right) \mathrm{d} x \\
\leq & c\left(f_{2 B} \exp \left(\beta g^{d}\right) \mathrm{d} x\right)\left(f_{2 B} f^{d} \mathrm{~d} x\right) \\
& +c f_{2 B} h^{d} \log ^{c_{0} \beta}\left(e+\frac{h}{\|f\|_{d, 2 B}}\right) \mathrm{d} x,
\end{aligned}
$$

where $c=c(n, d, \beta, C)>0$ and $\|f\|_{d, 2 B}=\left(f_{2 B} f^{d} \mathrm{~d} x\right)^{1 / d}$.

Remark 3.1. Lemma 3.1 is not limited to the case of $n=2$ or $n=3$.

We shall apply Lemma 3.1 to suitable powers of the functions

$$
\begin{aligned}
H & :=\left[D_{\varepsilon}^{2} f(\cdot, \varepsilon(u))\left(\varepsilon\left(\partial_{k} u\right), \varepsilon\left(\partial_{k} u\right)\right)\right]^{\frac{1}{2}}, \\
\Gamma & :=1+|\varepsilon(u)|^{2},
\end{aligned}
$$

where $u$ denotes a strong solution of system (1.1), which was constructed in $₫ 2$, We recall that

$$
u \in W_{r, \text { loc }}^{2}\left(\Omega ; \mathbb{R}^{2}\right) \quad \text { for all } r<2
$$


(compare statement i) of Theorem 1.1 and Remark 1.1) and use the weak form of (1.1) in the same way as in BF2] (compare the calculations starting from (5) and ending up with (9) of that paper, where we must take $E(x)=x$ and the tensor field $S$ must be replaced by $T(x, \varepsilon)$; note also that, by (3.1), the arguments of [BF2] are valid under the present hypotheses (1.2) and (1.3) ) in order to get

$$
\begin{aligned}
\int_{\Omega} \eta^{2} \partial_{k} \sigma: \varepsilon\left(\partial_{k} u\right) \mathrm{d} x \\
\leq-2 \int_{\Omega} \eta \partial_{k} \sigma_{i j} \partial_{i} \eta \partial_{k}[u-Q x]^{j} \mathrm{~d} x+\int_{\Omega}[\nabla u] u \cdot \partial_{k}\left(\eta^{2} \partial_{k}[u-Q x]\right) \mathrm{d} x \\
\quad-\int_{\Omega} g \cdot \partial_{k}\left(\eta^{2} \partial_{k}[u-Q x]\right) \mathrm{d} x+2 \int_{\Omega} \partial_{k} \pi \eta \nabla \eta \cdot \partial_{k}[u-Q x] \mathrm{d} x,
\end{aligned}
$$

where $\eta \in C_{0}^{\infty}(\Omega), 0 \leq \eta \leq 1, Q \in \mathbb{R}^{2 \times 2}$, and $\sigma:=D_{\varepsilon} f(\cdot, \varepsilon(u))$. Note that (3.2) exactly corresponds to inequality (9) of [BF2]. Of course, we may also use the " $\delta$-version" of (3.2) (see inequality (2.2) and the beginning of the proof of Lemma 2.4) together with the a priori estimates of $₫ 2$ (see Lemmas 2.3 and 2.5) to obtain (3.2) after passing to the limit as $\delta \rightarrow 0$. We fix some subdomain $\Omega^{\prime} \Subset \Omega$ and consider a disk $B_{2 r} \subset \Omega^{\prime}$. Moreover, assume that spt $\eta \subset B_{2 r}, \eta \equiv 1$ on $B_{r}$ and $|\nabla \eta| \leq c / r$. Using the fact that $u \in L^{\infty}\left(\Omega^{\prime} ; \mathbb{R}^{2}\right)$ (see (3.1)), from (3.2) we deduce the following estimate with a constant $c$ independent of $B_{2 r}$ :

$$
\begin{aligned}
& f_{B_{2 r}} \eta^{2} \partial_{k} \sigma: \varepsilon\left(\partial_{k} u\right) \mathrm{d} x \\
& \leq c\left[f_{B_{2 r}} \eta|\nabla \sigma||\nabla \eta||\nabla u-Q| \mathrm{d} x+f_{B_{2 r}}|\nabla u| \eta^{2}\left|\nabla^{2} u\right| \mathrm{d} x\right. \\
& \quad+f_{B_{2 r}} \eta|\nabla \eta||\nabla u||\nabla u-Q| \mathrm{d} x+f_{B_{2 r}}|g| \eta^{2}\left|\nabla^{2} u\right| \mathrm{d} x \\
& \left.\quad+f_{B_{2 r}}|g| \eta|\nabla \eta||\nabla u-Q| \mathrm{d} x+f_{B_{2 r}}|\nabla \pi| \eta|\nabla \eta||\nabla u-Q| \mathrm{d} x\right],
\end{aligned}
$$

where $|\nabla \pi|$ can be estimated via (1.1). As usual, this implies that

$$
|\nabla \pi| \leq|g|+|u||\nabla u|+|\nabla \sigma| .
$$

Thus, we get (recall that $g \in L^{\infty}$ )

$$
\begin{aligned}
& f_{B_{2 r}} \eta^{2} \partial_{k} \sigma: \varepsilon\left(\partial_{k} u\right) \mathrm{d} x \\
& \leq c\left[f_{B_{2 r}} \eta|\nabla \sigma||\nabla \eta||\nabla u-Q| \mathrm{d} x\right. \\
& \quad+f_{B_{2 r}}|\nabla u| \eta^{2}\left|\nabla^{2} u\right| \mathrm{d} x+f_{B_{2 r}} \eta|\nabla \eta||\nabla u||\nabla u-Q| \mathrm{d} x \\
& \left.\quad+f_{B_{2 r}} \eta^{2}\left|\nabla^{2} u\right| \mathrm{d} x+f_{B_{2 r}} \eta|\nabla \eta||\nabla u-Q| \mathrm{d} x\right] .
\end{aligned}
$$

As outlined in the proof of Lemma 2.2 we have the following counterpart of (2.3):

$$
\varepsilon\left(\partial_{k} u\right): \partial_{k} \sigma \geq \frac{1}{2} H^{2}-c \Gamma^{\frac{2 \bar{q}-p}{2}} .
$$

Furthermore, we observe that the inequality before (2.4) now reads as

$$
|\nabla \sigma|^{2} \leq H\left[D_{\varepsilon}^{2} f(\cdot, \varepsilon(u))\left(\partial_{k} \sigma, \partial_{k} \sigma\right)\right]^{\frac{1}{2}}+c \Gamma^{\frac{\bar{q}-1}{2}}|\nabla \sigma|
$$


which, by the second inequality of (1.2), implies the estimate

$$
|\nabla \sigma| \leq c H \tilde{h}+c \Gamma^{\frac{\bar{q}-1}{2}}
$$

with $\tilde{h}:=\max \left\{\Gamma^{(\bar{q}-2) / 4}, \Gamma^{(2-p) / 4}\right\}$. We return to (3.3) and make use of (3.4) and (3.5) in order to get the following inequality:

$$
\begin{aligned}
f_{B_{2 r}} & \eta^{2} H^{2} \mathrm{~d} x \\
\leq c & {\left[f_{B_{2 r}} \eta^{2} \Gamma^{\frac{2 \bar{q}-p}{2}} \mathrm{~d} x+f_{B_{2 r}} \eta|\nabla \eta| H \tilde{h}|\nabla u-Q| \mathrm{d} x\right.} \\
& +f_{B_{2 r}} \Gamma^{\frac{\bar{q}-1}{2}} \eta|\nabla \eta||\nabla u-Q| \mathrm{d} x+f_{B_{2 r}} \eta^{2}|\nabla u|\left|\nabla^{2} u\right| \mathrm{d} x \\
& \left.+f_{B_{2 r}} \eta|\nabla \eta||\nabla u||\nabla u-Q| \mathrm{d} x+f_{B_{2 r}} \eta^{2}\left|\nabla^{2} u\right| \mathrm{d} x+f_{B_{2 r}} \eta|\nabla \eta||\nabla u-Q| \mathrm{d} x\right] \\
=: & c \sum_{i=1}^{7} I_{i} .
\end{aligned}
$$

We estimate the terms on the right-hand side of (3.6) in accordance with ideas presented in [BFZ, by using $\left|\nabla^{2} u\right| \leq c|\nabla \varepsilon(u)| \leq c H \tilde{h}$ and the Sobolev-Poincaré inequality. For $\gamma:=4 / 3$, under an appropriate choice of $Q$ we obtain

$$
\begin{aligned}
I_{2} & \leq c r^{-1} f_{B_{2 r}} H \tilde{h}|\nabla u-Q| \mathrm{d} x \\
& \leq c\left[f_{B_{2 r}}(H h)^{\gamma} \mathrm{d} x\right]^{\frac{1}{\gamma}} \frac{1}{r}\left[f_{B_{2 r}}|\nabla u-Q|^{4} \mathrm{~d} x\right]^{\frac{1}{4}} \\
& \leq c\left[f_{B_{2 r}}(H \tilde{h})^{\gamma} \mathrm{d} x\right]^{\frac{2}{\gamma}} .
\end{aligned}
$$

Hölder's and Sobolev-Poincaré's inequalities give

$$
\begin{aligned}
I_{3} & \leq \frac{c}{r} f_{B_{2 r}}|\nabla u-Q| \Gamma^{\frac{\bar{q}-1}{2}} \mathrm{~d} x \leq \frac{c}{r}\left[f_{B_{2 r}}|\nabla u-Q|^{4} \mathrm{~d} x\right]^{\frac{1}{4}}\left[f_{B_{2 r}} \Gamma^{\gamma \frac{\bar{q}-1}{2}} \mathrm{~d} x\right]^{\frac{1}{\gamma}} \\
& \leq c\left[f_{B_{2 r}}\left|\nabla^{2} u\right|^{\gamma} \mathrm{d} x\right]^{\frac{1}{\gamma}}\left[f_{B_{2 r}} \Gamma^{\gamma^{\frac{\bar{q}-1}{2}}} \mathrm{~d} x\right]^{\frac{1}{\gamma}} \\
& \leq c\left[f_{B_{2 r}}(H \tilde{h})^{\gamma} \mathrm{d} x\right]^{\frac{1}{\gamma}}\left[f_{B_{2 r}} \Gamma^{\bar{q}-1} \mathrm{~d} x\right]^{\frac{1}{2}} \\
& \leq c\left[f_{B_{2 r}}(H \tilde{h})^{\gamma} \mathrm{d} x\right]^{\frac{2}{\gamma}}+c f_{B_{2 r}} \Gamma^{\bar{q}-1} \mathrm{~d} x .
\end{aligned}
$$

$I_{4}$ is estimated as follows:

$$
\begin{aligned}
I_{4} & \leq c f_{B_{2 r}} H \tilde{h}|\nabla u| \mathrm{d} x \leq c\left[f_{B_{2 r}}(H \tilde{h})^{\gamma} \mathrm{d} x\right]^{\frac{1}{\gamma}}\left[f_{B_{2 r}}|\nabla u|^{4} \mathrm{~d} x\right]^{\frac{1}{4}} \\
& \leq c\left[f_{B_{2 r}}(H \tilde{h})^{\gamma} \mathrm{d} x\right]^{\frac{2}{\gamma}}+c\left[f_{B_{2 r}}|\nabla u|^{4} \mathrm{~d} x\right]^{\frac{1}{2}},
\end{aligned}
$$


where the last term is bounded from above by $\int_{B_{2 r}} \tilde{\Gamma}^{2} \mathrm{~d} x, \tilde{\Gamma}:=1+|\nabla u|^{2}$. Next, we observe that

$$
\begin{aligned}
I_{5} & \leq \frac{c}{r}\left[f_{B_{2 r}}|\nabla u-Q|^{4} \mathrm{~d} x\right]^{\frac{1}{4}}\left[f_{B_{2 r}}|\nabla u|^{\gamma} \mathrm{d} x\right]^{\frac{1}{\gamma}} \\
& \leq c\left[f_{B_{2 r}}\left|\nabla^{2} u\right|^{\gamma} \mathrm{d} x\right]^{\frac{1}{\gamma}}\left[f_{B_{2 r}}|\nabla u|^{\gamma} \mathrm{d} x\right]^{\frac{1}{\gamma}} \\
& \leq c\left[f_{B_{2 r}}(H \tilde{h})^{\gamma} \mathrm{d} x\right]^{\frac{2}{\gamma}}+c f_{B_{2 r}} \tilde{\Gamma} \mathrm{d} x,
\end{aligned}
$$

for $I_{6}$ we have

$$
\begin{aligned}
I_{6} & \leq c f_{B_{2 r}} H \tilde{h} \mathrm{~d} x \leq c\left[f_{B_{2 r}}(H \tilde{h})^{\gamma} \mathrm{d} x\right]^{\frac{1}{\gamma}}\left[f_{B_{2 r}} \mathrm{~d} x\right]^{\frac{1}{4}} \\
& \leq c\left[f_{B_{2 r}}(H \tilde{h})^{\gamma} \mathrm{d} x\right]^{\frac{2}{\gamma}}+c f_{B_{2 r}} \Gamma \mathrm{d} x,
\end{aligned}
$$

and finally we see that

$$
\begin{aligned}
I_{7} & \leq \frac{c}{r} f_{B_{2 r}}|\nabla u-Q| \mathrm{d} x \leq c\left[f_{B_{2 r}}\left|\nabla^{2} u\right|^{\gamma} \mathrm{d} x\right]^{\frac{1}{\gamma}}\left[f_{B_{2 r}} \mathrm{~d} x\right]^{\frac{1}{\gamma}} \\
& \leq c\left[f_{B_{2 r}}(H \tilde{h})^{\gamma} \mathrm{d} x\right]^{\frac{2}{\gamma}}+f_{B_{2 r}} \Gamma \mathrm{d} x .
\end{aligned}
$$

Collecting terms, from (3.6) we deduce that

$$
\begin{array}{r}
f_{B_{r}} H^{2} \mathrm{~d} x \leq c\left[f_{B_{2 r}} G \mathrm{~d} x+\left[f_{B_{2 r}}(H \tilde{h})^{\gamma} \mathrm{d} x\right]^{\frac{2}{\gamma}}\right], \\
G:=\max \left\{\tilde{\Gamma}^{2}, \tilde{\Gamma}^{\bar{q}-1}, \tilde{\Gamma}^{\frac{2 \bar{q}-p}{2}}\right\} ;
\end{array}
$$

thus

$$
\left[f_{B_{r}} H^{2} \mathrm{~d} x\right]^{\frac{\gamma}{2}} \leq c f_{B_{2 r}}(H \tilde{h})^{\gamma} \mathrm{d} x+c\left[f_{B_{2 r}} G \mathrm{~d} x\right]^{\frac{\gamma}{2}} .
$$

Now we would like to apply Lemma 3.1 with the choices

$$
d=\frac{2}{\gamma}=\frac{3}{2}, f=H^{\gamma}, g=\tilde{h}^{\gamma}, h=G^{\frac{2}{3}} .
$$

For this purpose, we claim that

$$
\exp \left(\beta g^{d}\right)=\exp \left(\beta \tilde{h}^{2}\right) \in L_{\text {loc }}^{1}(\Omega)
$$

for any number $\beta>0$. Indeed, the calculations in the proof of Lemma 2.5 (combine the estimate for $T_{2}$ with the information that $\nabla u_{\delta} \in L_{\text {loc }}^{r}\left(\Omega ; \mathbb{R}^{2 \times 2}\right)$ for any $\left.r<\infty\right)$ imply $\Phi:=\Gamma^{p / 4} \in W_{2, \text { loc }}^{1}(\Omega)$; hence, by Trudinger's inequality (see Theorem 7.16 of [GT]), we have

$$
\int_{B_{\rho}} \exp \left(\beta_{0} \Phi^{2}\right) \mathrm{d} x \leq c(\rho)<\infty
$$

for any disk $B_{\rho} \Subset \Omega$. Here $\beta_{0}$ depends on the $W_{2}^{1}$-bound of $\Phi$ on $B_{\rho}$. In particular, we see that

$$
\int_{B_{\rho}} \exp \left(\beta \Phi^{2-\kappa}\right) \mathrm{d} x \leq c(\rho, \beta, \kappa)<\infty
$$


for any $0<\kappa<1$ and all $\beta>0$. Since $\bar{q}<p+2$, we have the inequality

$$
\Gamma^{\frac{\bar{q}-2}{2}} \leq \Gamma^{\frac{p}{4}(2-\kappa)}=\Phi^{2-\kappa}
$$

for some small $\kappa>0$, and clearly (since $p>1$ )

$$
\Gamma^{\frac{2-p}{2}} \leq \Phi^{2-\kappa},
$$

so that $\tilde{h}^{2} \leq \Phi^{2-\kappa}$. Thus, (3.7) follows from (3.8).

Moreover, since $f^{d}=D_{\varepsilon}^{2} f(\cdot, \varepsilon(u))\left(\partial_{k} \varepsilon(u), \partial_{k} \varepsilon(u)\right)$, the fact that $f^{d} \in L_{\text {loc }}^{1}(\Omega)$ is deduced from Lemma 2.4 together with the uniform local estimates for the right-hand side. The statement $h \in L_{\text {loc }}^{d}(\Omega)$ is immediate by the definition of $G$. Now, the lemma implies that

$$
\int_{B_{\rho}} H^{2} \log ^{c_{0} \beta}(e+H) \mathrm{d} x \leq c(\beta, \rho)
$$

for all disks $B_{\rho} \subset \Omega^{\prime}$ and all $\beta>0$. Recall that (see (3.5))

$$
|\nabla \sigma| \leq c H \tilde{h}+c \Gamma^{\frac{\bar{q}-1}{2}}
$$

and that

$$
\int_{B_{\rho}}\left(\Gamma^{\frac{\bar{q}-1}{2}}\right)^{2} \log ^{c_{0} \beta}\left(e+\Gamma^{\frac{\bar{q}-1}{2}}\right) \mathrm{d} x \leq c(\beta, \rho) .
$$

The same is true for the function $H \tilde{h}$. To verify this statement, we recall the following elementary inequality (see (2.12) in [BFZ]). Let $a, b \geq 0$. Then, for any $\alpha>0$, there is a constant $c(\alpha)>0$ such that

$$
(a b)^{2} \log ^{\alpha}(e+a b) \leq 2^{\alpha} a^{2} \log ^{\alpha+2}(e+a)+c(\alpha) \exp (6 b) .
$$

Applying (3.9) to $a=H, b=\tilde{h}$, we obtain the desired estimate:

$$
\int_{B_{\rho}}\left((H \tilde{h})^{\frac{\bar{q}-1}{2}}\right)^{2} \log ^{c_{0} \beta}\left(e+(H \tilde{h})^{\frac{\bar{q}-1}{2}}\right) \mathrm{d} x \leq c(\beta, \rho) .
$$

Now, using the estimate for $|\nabla \sigma|$ combined with the inequality

$$
(a+b)^{2} \log ^{\alpha}(e+a+b) \leq c\left[a^{2} \log ^{\alpha}(e+a)+b^{2} \log ^{\alpha}(e+b)\right],
$$

we finally arrive at

$$
\int_{B_{\rho}}|\nabla \sigma|^{2} \log ^{c_{0} \beta}(1+|\nabla \sigma|) \mathrm{d} x \leq c(\beta, \rho)<\infty
$$

for any $\beta>0$. This shows that $\sigma \in C^{0}\left(\Omega ; \mathbb{S}^{n}\right)$, by quoting Example 5.3 of $[\mathrm{KKM}$. Now $u \in C^{1, \alpha}\left(\Omega ; \mathbb{R}^{2}\right)$ follows as outlined in [BF2, p. 1616].

\section{REFERENCES}

[AM] E. Acerbi and G. Mingione, Regularity results for stationary electrorheological fluids, Arch. Rational Mech. Anal. 164 (2002), 213-259. MR1930392 (2003g:35020)

[ABF] D. Apushkinskaya, M. Bildhauer, and M. Fuchs, Steady states of anisotropic generalized Newtonian fluids, J. Math. Fluid Mech. 7 (2005), 261-297. MR2177129 (2006g:35200)

[BF1] M. Bildhauer and M. Fuchs, Variants of the Stokes problem: The case of anisotropic potentials, J. Math. Fluid Mech. 5 (2003), 364-402. MR2004292 (2004g:76045)

[BF2] - A regularity result for stationary electrorheological fluids in two dimensions, Math. Methods Appl. Sci. 27 (2004), 1607-1617. MR2077446 (2005f:35253)

[BF3] — Regularization of convex variational problems with applications to generalized Newtonian fluids, Arch. Math. (Basel) 84 (2005), 155-170. MR2120709 (2005m:49057)

[BF4] - $C^{1, \alpha}$-solutions to non-autonomous anisotropic variational problems, Calc. Var. 24 (2005), 309-340. MR2174429(2006k:49101) 
[BFZ] M. Bildhauer, M. Fuchs, and X. Zhong, A lemma on the higher integrability of functions with applications to the regularity theory of two-dimensional generalized Newtonian fluids, Manuscripta Math. 116 (2005), 135-156. MR2122416 (2006d:35191)

[DER] L. Diening, F. Ettwein, and M. Růžička, $C^{1, \alpha}$-regularity for electrorheological fluids in two dimensions, Preprint Univ. Freiburg, 2004.

[ELM] L. Esposito, F. Leonetti, and G. Mingione, Sharp regularity for functionals with $(p, q)$ growth, J. Differential Equations 204 (2004), 5-55. MR2076158 (2005e:49002)

[E] F. Ettwein, Elektrorheologische Flüssigkeiten: Existenz starker Lösungen in zweidimensionalen Gebieten, Diplomarbeit, Univ. Freiburg, 2002.

[ER] F. Ettwein and M. Růžička, Existence of strong solutions for electrorheological fluids in two dimensions: Steady Dirichlet problem, Geometric Analysis and Nonlinear Partial Differential Equations, Springer-Verlag, Berlin, 2003, pp. 591-602. MR2008358 (2004i:76195)

[FMS] J. Frehse, J. Málek, and M. Steinhauer, On analysis of steady flows of fluids with shear-dependent viscosity based on the Lipschitz truncation method, SIAM J. Math. Anal. 34 (2003), 1064-1083. MR.2001659 (2005c:76007)

[Ga] G. Galdi, An introduction to the mathematical theory of the Navier-Stokes equations. Vol. 1, Springer Tracts Nat. Philos., vol. 38, Springer-Verlag, New York, 1994. MR.1284205 (95i:35216a)

[Gi] M. Giaquinta, Multiple integrals in the calculus of variations and nonlinear elliptic systems, Ann. of Math. Stud., vol. 105, Princeton Univ. Press, Princeton, NJ, 1983. MR717034 (86b:49003)

[GT] D. Gilbarg and N. Trudinger, Elliptic partial differential equations of the second order, 2nd ed., Grundlehren Math. Wiss., vol. 224, Springer-Verlag, Berlin, 1983. MR737190 (86c:35035)

[KMS] P. Kaplický, J. Málek, and J. Stará, $C^{1, \alpha}$-solutions to a class of nonlinear fluids in two dimensions - stationary Dirichlet problem, Zap. Nauchn. Sem. S.-Peterburg. Otdel. Mat. Inst. Steklov. (POMI) 259 (1999), 89-121; English transl., J. Math. Sci. (N.Y.) 109 (2002), no. 5, 1867-1893. MR 1754359 (2001f:35329)

[KKM] J. Kauhanen, P. Koskela, and J. Malý, On functions with derivatives in a Lorentz space, Manuscripta Math. 100 (1999), 87-101. MR1714456 (2000j:46064)

[La] O. A. Ladyzhenskaya, Mathematical questions of the dynamics of a viscous incompressible fluid, 2nd ed., "Nauka", Moscow, 1970; English transl. of 1st ed., The mathematical theory of viscous incompressible flow, Math. Appl., vol. 2, Gordon and Breach, New York, 1969. MR0271559 (42:6442) MR0254401(40:7610)

[Pi] K. I. Piletskas, Three-dimensional solenoidal vectors, Zap. Nauchn. Sem. Leningrad. Otdel. Mat. Inst. Steklov. (LOMI) 96 (1980), 237-239; English transl., J. Soviet Math. 21 (1983), no. 5, 821-823. MR579486 (81k:46035)

[R] M. Růžička, Electrorheological fluids: Modeling and mathematical theory, Lecture Notes in Math., vol. 1748, Springer-Verlag, Berlin, 2000. MR1810360 (2002a:76004)

[Z] V. V. Zhikov, On Lavrentiev's phenomenon, Russian J. Math. Phys. 3 (1995), 249-269. (English) MR1350506 (96h:49019)

Department of Mathematics, Saarland University, P.O. Box 1511 50, D-66041 Saarbrücken, GERMANY

E-mail address: bibi@math.uni-sb.de

Department of Mathematics, Saarland University, P.O. Box 1511 50, D-66041 Saarbrǘcken, GERMAnY

E-mail address: fuchs@math.uni-sb.de

Department of Mathematics and Statistics, University of Jyväskylä, P.O. Box 35, FiN40014 University of Jyväskylä, FinLAND

E-mail address: zhong@maths.jyu.fi

Received 31/OCT/2005

Originally published in English 